\title{
Reply to Dunning
}

To THE EDitor-The commentary by Dunning helpfully raises the question about the sensitivity of estimation of the vaccine efficacy (VE) curve to the choice of the baseline immunogenicity predictor (BIP) that is used. In the Zostavax Efficacy and Safety Trial (ZEST) example in our article the VE curve is estimated as a function of 2 binding antibody markers: fold rise and week 6 titer, in each case using baseline titer as the BIP [1]. We apply several statistical methods for estimating the VE curve, each of which separately estimates 2 terms: the disease risk 
in vaccine recipients conditional on the marker and the disease risk in placebo recipients conditional on the marker, which for a placebo recipient is the marker that s/he would have had if, contrary to fact, $\mathrm{s} /$ he had been assigned to receive the vaccine. We call these risks $\operatorname{Vrisk}(s)$ and $\operatorname{Prisk}(s)$, respectively, for subgroups with marker level $s$, where the VE curve is $\operatorname{VE}(s)=1-\operatorname{Vrisk}(s) / \operatorname{Prisk}(s)$, which measures VE ranging over subgroups defined by the marker level $s$. Vrisk(s) can be straightforwardly estimated using regression statistical methods without the need for any BIP, because the marker is observed in vaccinated individuals, such that the key issue is estimation of Prisk(s). In our Discussion, we noted that if $\operatorname{Vrisk}(s)$ and $\operatorname{Prisk}(s)$ are correctly modeled such that valid estimates are obtained, then the VE curve is accurately (unbiasedly) estimated, and this statement holds for any BIP that is reasonably well correlated with the marker, as it is for the ZEST example [1]. Therefore, we recast the question raised by Dunning as "How does a scientist jointly select the BIP and a model for Prisk $(s)$ to obtain a valid estimate of $\operatorname{Prisk}(s)$, and how can one evaluate the validity?"

Because the markers are missing in placebo recipients, the problem of modeling and estimation of $\operatorname{Prisk}(s)$ fits into the field of statistical methods for handling missing data. As for all problems in this field, modeling assumptions must be made to obtain valid estimates, and it behooves the scientist to: (1) transparently list all modeling assumptions, (2) empirically check all modeling assumptions based on the observed data to the extent possible, (3) acknowledge where validity of the methods depends on assumptions that cannot be empirically verified, and (4) conduct sensitivity analyses to check the sensitivity of the estimates to departures from the untestable assumptions [2]. These steps are salient for the VE curve problem.

Multiple imputation methods constitute a useful framework for understanding the requirements of VE curve estimation. In multiple imputation, parti- cipant characteristics not necessarily of scientific interest in themselves are used for the technical purpose of filling in missing values, and the scientist seeks the best available predictors (participant characteristics) and best-fitting imputation models, and the accuracy and precision of the estimation of interest depends on the success of these tasks. Similarly, for the VE curve problem, the scientist seeks the most predictive BIPs (as a technical device) and the best-fitting imputation models, and the accuracy and precision of estimation of the VE curve depend on the success of these tasks. The fact that the performance of VE curve estimation depends on the quality of the available technical tools is normal science.

We now address the question of how to seek assurance that the selected BIP and model for Prisk $(s)$ yield a valid estimate of Prisk $(s)$. The "closeout placebo vaccination" (CPV) technique [3] can be very helpful for providing this assurance because it provides a way to test the validity of modeling assumptions for $\operatorname{Prisk}(s)$, and is thus recommended (CPV crosses over placebo recipients at the end of study follow-up to receive the vaccine and have the marker directly measured). However, if CPV is not performed, there is no way, to our knowledge, that model fit for Prisk(s) can be fully tested without making additional assumptions.

Beyond our general recommendation to follow the 4 steps listed above, for a given efficacy trial, context-specific knowledge is needed to posit a credible BIP and model for Prisk(s); many approaches have been suggested. For example, Follmann [3] showed that Prisk $(s)$ can be accurately estimated if the Prisk $(s)$ model is correctly specified and the BIP used does not affect disease after accounting for the marker. This may be achieved through "baseline irrelevant vaccination," wherein a vaccine different from the one in the trial is given to both vaccine and placebo recipients and an immune response to this unrelated vaccine is used as the BIP. Selecting a baseline vaccine with no cross-reactivity with the vaccine under study could potentially achieve this objective. A BIP that does not affect disease after accounting for the marker can be thought of as an "instrument" for the marker; the statistics and econometrics literatures include many examples of estimation via instrumental variables. A second approach includes a set of baseline participant characteristics in the Prisk(s) model, such that after accounting for these characteristics the marker does not affect disease risk. A third approach, which applies for a binary marker, allows the placebo disease risk to depend arbitrarily on the marker and adds the assumption that the placebo group disease risk during discrete follow-up intervals is constant over time [4].

If multiple BIPs strongly predictive of the marker are available, then the above research process may be undertaken, accounting for those multiple BIPs. Similar estimated VE curves using different BIPs would provide reassurance that the results are robust to the choice of BIP. On the other hand, if the estimated VE curves vary depending on the BIP, then more caution in interpretation is warranted. Moreover, multiple BIPs may be combined to improve prediction over a single BIP, and VE curve estimation incorporating multiple BIPs is an active area of research. In the ZEST example, only one strongly predictive BIP was available.

We close by responding to 2 other points in the commentary. First, Dunning states that an increasing VE curve in the fold-rise marker is "guaranteed" because the BIP is negatively correlated with both disease risk and with the marker. This guarantee is based on a supposed transitivity property of correlation, which would state that for 3 random variables, $x, y$, and $z$, negative correlation between $x$ and $y$ and between $x$ and $z$ implies positive correlation between $y$ and $z$. However, this transitivity property for correlation is not true [5], so there is no guarantee. Second, Dunning focuses on how the relationship between the selected BIP and placebo group disease risk affects the VE curve estimation. However, because 
the Prisk(s) modeling always conditions on the marker, the salient issue is instead this relationship conditional on the marker. Therefore, the association of the selected BIP with disease does not drive the estimated VE curve. Rather, the VE curve may be accurately estimated for many different types of associations of the BIP with disease, and the BIP should be viewed as a technical device with purpose to impute the marker for placebo recipients.

\section{Note}

Potential conflicts of interest. All authors: No potential conflicts.

All authors have submitted the ICMJE Form for Disclosure of Potential Conflicts of Interest. Conflicts that the editors consider relevant to the content of the manuscript have been disclosed.
Peter B. Gilbert, ${ }^{1}$ Erin E. Gabriel, ${ }^{3}$ Michael G. Hudgens, ${ }^{4}$ Xiaopeng Miao, ${ }^{5}$ Xiaoming Li, ${ }^{2}$ Shu-Chih Su, ${ }^{6}$ Janie Parrino, ${ }^{6}$ and Ivan S. F. Chan

${ }^{1}$ Vaccine and Infectious Disease Division, Fred Hutchinson Cancer Research Center and Department of Biostatistics, University of Washington, and ${ }^{2}$ Biostatistics, Gilead Sciences, Seattle,

Washington; ${ }^{3}$ National Institute of Allergy and Infectious Diseases, Biostatistics Research Branch, Bethesda, Maryland; ${ }^{4}$ Department of Biostatistics, University of North Carolina at Chapel Hill; ${ }^{5}$ Biostatistics, Biogen, Cambridge, Massachusetts; and

${ }^{6}$ Merck \& Co, Whitehouse Station, New Jersey

\section{References}

1. Gilbert PB, Gabriel EE, Miao X, et al. Fold rise in antibody titers by measured by glycoprotein-based enzyme-linked immunosorbent assay is an excellent correlate of protection for a herpes zoster vaccine, demonstrated via the vaccine efficacy curve. J Infect Dis 2014; 210:1573-81.
2. Little RJ, D'Agostino R, Cohen ML, et al. The prevention and treatment of missing data in clinical trials. N Engl J Med 2012; 367:1355-60.

3. Follmann D. Augmented designs to assess immune response in vaccine trials. Biometrics 2006; 62:1161-9.

4. Long D, Hudgens MG, Wu C-D. Surrogates of protection in repeated low-dose challenge experiments. Stat Med 2015; 34:1747-60.

5. Langford E, Schwertman N, Owens M. Is the property of being positively correlated transitive? Am Stat 2001; 55:322-5.

Received 8 May 2015; accepted 11 May 2015; electronically published 17 May 2015.

Correspondence: Peter B. Gilbert, PhD, Vaccine and Infectious Disease Division, Fred Hutchinson Cancer Research Center, 1100 Fairview Ave N, P0 Box 19024, Seattle, WA 98109 (pgilber!@scharp.org).

The Journal of Infectious Diseases ${ }^{\circledR} \quad$ 2015;212:1521-3 (C) The Author 2015. Published by Oxford University Press on behalf of the Infectious Diseases Society of America. All rights reserved. For Permissions, please e-mail: journals. permissions@oup.com.

DOl: 10.1093/infdis/jiv287 